\title{
Towards Co-evolution of Information, Life and Artificial Life
}

\author{
Masami Hagiya and Ibuki Kawamata \\ Department of Computer Science, \\ Graduate School of Information Science and Technology, \\ The University of Tokyo 7-3-1 Hongo, Bunkyo-ku, Tokyo, 113-8656, Japan \\ \{hagiya, ibuki\}@is.s.u-tokyo.ac.jp
}

\begin{abstract}
We will begin with a simplified view of systems biology and synthetic biology. Systems biology extracts information from life, while synthetic biology converts information to reality. This cycle allows the coevolution of life and information, and accelerates the evolution of both. Additionally, the field of molecular robotics has recently emerged. This field is attempting to implement artificial life using biological molecules. We foresee that molecular robots will interface information and life, and the distinction among information, life and artificial life will eventually become a blur. Once molecular robots gain the ability to evolve, then co-evolution of the three will lead to a new stage of intelligence.
\end{abstract}

\section{Introduction}

We are currently in an era in which life and information can evolve together. Among the fields of research that involve both information technology and biology, bioinformatics and systems biology analyze "big data" obtained by cutting-edge bio-imaging technologies [8], and construct information (models and databases) on life on top of computers and networks, recently described as the "cloud". Conversely, synthetic biology, another emerging field, attempts to construct reality from models designed by humans [7. If systems biology is regarded as reverse engineering of life, then synthetic biology advocates forward engineering of life. These research fields thus form a cycle of life and information, as described in Figure 1 .

The implication of this cycle is profound. Life has evolved for billions of years and adapted to the environments of the earth. This is a type of "optimization", which consists of extremely complex processes. In addition, components at a lower level of hierarchy of life self-organize and express behaviors at an advanced level; this phenomenon is called "emergence". Based on this cycle, evolution and self-organization of life can be replaced with evolution and self-organization of information. Furthermore, co-evolution of life and information is possible. This situation is depicted in Figure 2 In addition to the original route, there exists another route for evolution and self-organization in life, where life is first converted to information, which evolves and self-organizes, and is then converted back to life. It is important to note that many existing methods for evolution and 


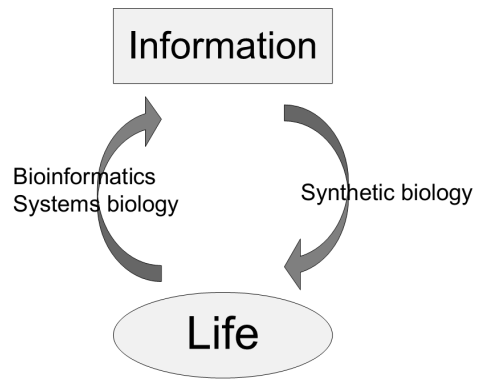

Fig. 1. Cycle around life and information

self-organization of information, such as evolutionary computation, are borrowed or inspired by evolution and the self-organization of life. The two routes may act in parallel to create new kinds of life. As a result, the evolution of life is accelerated compared with the situation where the two routes are independent, because a single step of evolution in one route may trigger a few steps of evolution in the other and result in exponential growth in the sense of singularity.

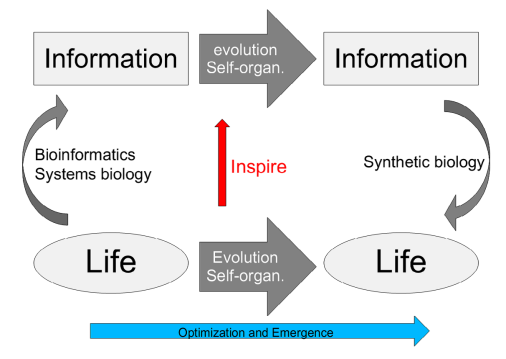

Fig. 2. CyCo-evolution of life and information

In this position paper, we will first describe the spectrum of wet artificial life, including synthetic biology and molecular robotics, in Section 2, Research fields in this spectrum attempt to construct complex artificial systems from molecules; in particular, biomolecules such as DNA and protein. According to synthetic biology, components from life can be borrowed and modified. Some research fields, such as molecular robotics, attempt to construct life-like systems from basic components. However, these research fields share a single goal. In Section 3 , the evolution and self-organization of information are briefly sketched. We classify them into two types: those by artificial intelligence and those by human intelligence, and put them in the context of the cloud, where various types of intelligence cooperate. We then envision the future from the perspective of molecular robotics in Section 4 . We foresee that molecular robots will interface information and life, and the distinction among information, life and artificial 
life will eventually blur. After molecular robots gain the ability to evolve, coevolution of the three will lead to a new stage of intelligence.

\section{Spectrum of Wet Artificial Life}

Channon et al. summarized and classified the approaches to synthetic biology, as described in Figure 3, which is simplified from the original [2]. On the left of the figure, various levels in the biological hierarchy from simpler to more complex are shown, with examples. The x-axis denotes the "unnaturalness" of synthesized systems. This figure clearly shows the direction towards constructing more complex and unnatural systems starting from natural and simple ones. Needless to say, it covers more than synthetic biology in the narrow sense. For example, they include organic chemistry and supra-molecular chemistry. In particular, the emerging field of molecular robotics is introduced in the next section. The ultimate goal of these research fields is "encapsulated complex systems", as shown in Figure 3. The two arrows represent synthetic biology (in the narrow sense) and molecular robotics, respectively.

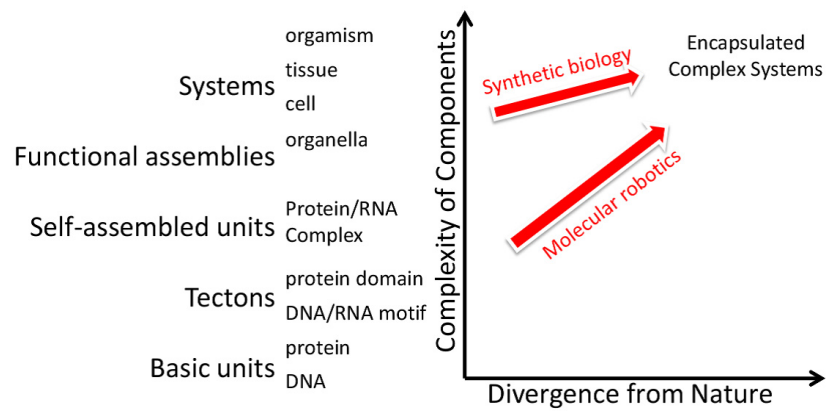

Fig. 3. Spectrum of synthetic biology

\subsection{Molecular Robotics}

Molecular robotics is an emerging research field whose aim is to construct autonomous systems from molecules. Autonomous systems suggest those composed of sensors, computers (information processing units) and actuators. Signals from the external environment are recognized by the sensors and transformed into internal signals fed to the information processing units, which perform computations and make judgments that order the actuator to perform its functions. In addition to these three components, an autonomous system should include encapsulating structures, as the phrase "encapsulated complex systems" in Figure 3 suggests. Energy sources that drive the three components are also necessary. 
Presently, molecular robotics utilizes mainly DNA molecules [1, which is why the field is also described as DNA robotics. There are many reasons for the use of DNA: DNA can form various structures in terms of hydrogen bonds between complementary sequences [10, which can be rationally designed and chemically synthesized; and DNA also allows various chemical modifications. Some sequences have chemical activity and can be used as sensors or actuators [3]. Therefore, molecular robots can be constructed using only DNA molecules. Such DNA robots may be inefficient for concrete applications, but are convenient for prototyping. Currently, an increasing number of chemists are joining the field, so additional types of molecules will likely be used in future applications.

\subsection{Synthetic Biology}

Cells possess all the necessary functions for autonomous systems. For example, membrane receptors are sensors. Information processing inside a cell is completed by genetic circuits, signal transduction pathways, etc. There are also several types of actuators within a cell. Protein synthesis per se is a type of actuation. Movement of a cell, such as chemotaxis, is also a type of actuation. In addition to these functions, cells themselves can also reproduce. Therefore, why not make robots by re-engineering cells? This idea has led to the emerging research field known as synthetic biology [7].

In synthetic biology, the international student competition known as the International Genetically Engineered Machine Competition (iGEM), is held annually and is becoming increasingly popular. Many teams of undergraduate students from all over the world congregate and present their ideas and experimental results.

As an emerging field, research in synthetic biology is presently very diverse. For example, various case studies are being conducted, including those presented at iGEM. Some case studies have specific applications, such as drug delivery and bio-fuel production. Efforts are also being made to introduce engineering disciplines into synthetic biology, such as standardization and abstraction of genetic components. Some researchers are interested in the construction of a minimal cell, which contains the minimum set of genes required for survival. Technology for the swapping of genomes in a cell facilitates production of a cell with a completely designed genome. Attempts are also being made to construct a cell from scratch. This is closely related to molecular robotics, and involves the technology required for generation of artificial membranes.

\section{Evolution and Self-organization of Information}

Evolution and self-organization of information is diverse. Here, they are classified into two types: those by human intelligence and those by artificial intelligence. Both have been amplified or powered by recent developments in information technology. Needless to say, these two types are not separate, but are interrelated. As we will discuss later, they even cooperate in the cloud. 


\subsection{Amplification of Human Intelligence}

Although the phrase Web 2.0 is becoming outdated, collaboration in the cloud is becoming more common, resulting in so-called collective intelligence. While Wikipedia is a typical example, synthetic biology is also a good example of collective intelligence. The organizers of iGEM are constructing a genetic component database (called parts) for use by participants of the competition, who should submit their parts to the database while being allowed to freely use all parts therein. The organizers thus accumulate knowledge for use in future applications, such as medicine, environment preservation and food production.

In general, collaboration in the cloud is becoming increasingly fine-grained and automated. In terms of wet experiments in synthetic biology, pieces of information that were typically kept in laboratory notebooks are now being stored in the cloud in a partially-automated fashion. This is in contrast to traditional biological databases in which results from a series of experiments were stored in conjunction with publications after completion of the related experiments.

We are currently developing a database for wet biological experiments and using it for our own experiments in the fields of synthetic biology and molecular robotics [4. This database is based on MediaWiki, a free, open-source wiki package originally developed for Wikipedia. A snapshot of a project page is shown in Figure 4. A project page describes a schedule of experimental steps, such as those beginning with plasmid preparation by infusion and ending with plasmid extraction by a miniprep procedure. A schedule of experimental steps is shown as a workflow graph, from which pages keeping information on materials and pages for concrete experiments are linked. We use MediaWiki templates extensively so


automatically generate a workflow graph and related material and experimental pages. The database can also be used with a tablet computer, such as an iPad, so that it replaces laboratory notebooks during wet experiments. We are also developing interfaces that control experimental devices to automate specification of parameters and extraction of data.

Using this database, we have conducted wet experiments in synthetic biology. They include almost all basic experimental steps in synthetic biology, including transformation, gel electrophoresis, photospectroscopy, infusion, mutagenesis, sequencing, minipreps, etc. We also performed experiments in which AND gates were implemented in Escherichia coli.

We hope that this database will eventually control robots and lead to full automation of wet experiments. Dry experiments, such as prediction of the energy parameters of DNA strands, can already be conducted using the database, and experimental results are automatically inserted therein. External services, such as DNA synthesis and plasmid sequencing, can also be linked from the database through the web interface. 


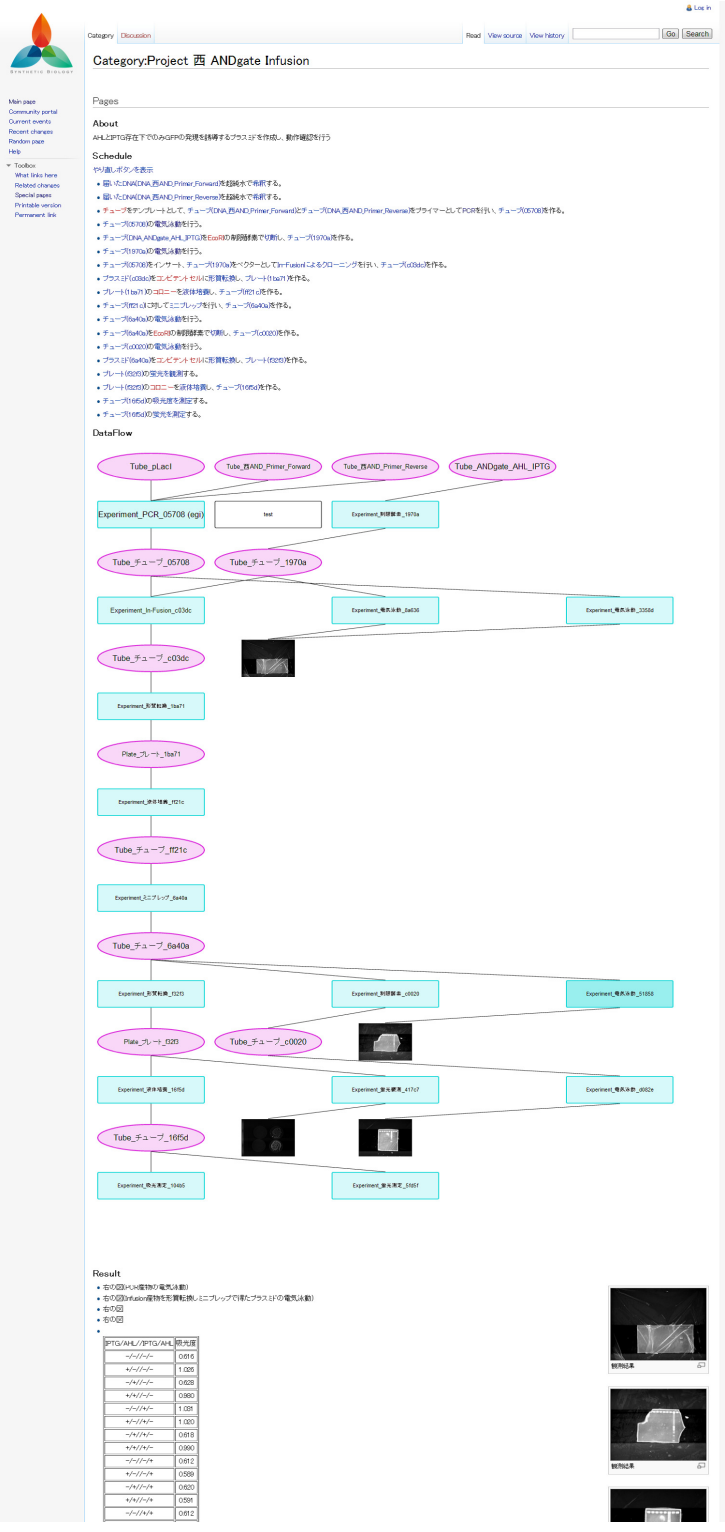

Fig. 4. A project page 


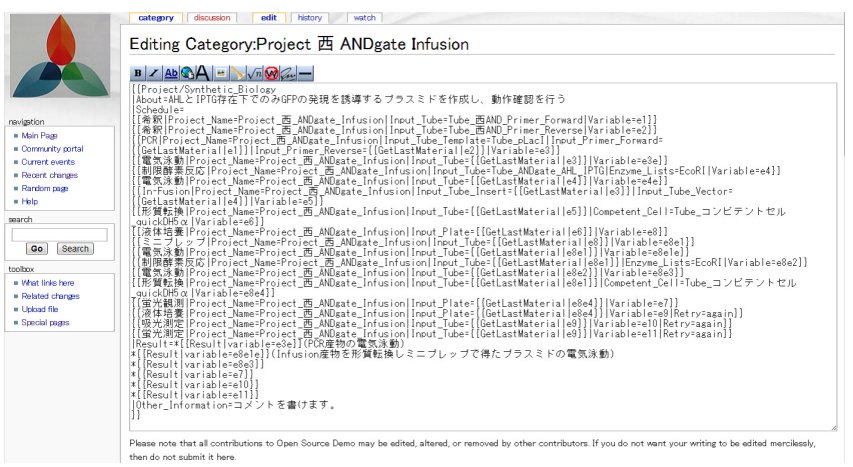

Fig. 5. Source code of the project page in Fig 4

\subsection{Automatic Design by Artificial Intelligence}

By artificial intelligence, we mean automatic design of systems, in general. For example, in the systems biology field, it is common to predict genetic circuits from microarray data. For this purpose, evolutionary computations, such as genetic algorithms and particle swarm optimization, are commonly used. In addition to predictions, they can also be used for the design and synthesis of molecular and cellular systems.

Evolutionary computation, the most typical of the so-called bio-inspired methods, borrows the ability of life to evolve and optimizes its functions with respect to its environment. For example, in genetic algorithms, evolution by mutation, crossover and selection are mimicked in silico to solve various search and optimization problems. Compared with other optimization methods, this is more effective when the search space is large and the evaluation function is complex, and it has been applied to various design problems, including those of DNA robots and artificial genetic circuits. In the latter applications, we can say that methods inspired from life create life!

A concrete example of applying evolutionary computation to molecular robotics can be found in the work by Kawamata et al. [5]. They employed simulated annealing to design some DNA devices including logic gates. A specification of a device is first given and DNA devices are randomly generated at the level of DNA segments consisting of ten to twenty bases. Generated devices are then simulated and evaluated with respect to the specification. Those devices with a good evaluation value are chosen and improved by random mutation.

It seems interesting to combine such in-silico evolution with in-vitro evolution of molecules. For example, in-silico evolution may also introduce artificial molecules having hypothetical functions. If a device containing such an artificial molecule has a high evaluation value, it is worth while to look for a real molecule having the specific function by in-vitro evolution. If new molecules are actually found by in-vitro evolution, they are added to the library of molecules for constructing molecular devices and increase the variety of possible devices. 


\subsection{Cloud}

Cloud computing started with shared computer resources, such as storage, CPU time and networks. As mentioned earlier, resources shared in the cloud are becoming increasingly fine-grained. For example, while entire virtual machines are shared in Amazon EC2, single files are shared in distributed storage systems such as Dropbox. In our database of biological experiments, we are also attempting to store and share in the cloud more fine-grained information regarding each experimental step.

In addition, an increasing number of services are connected to the cloud, including laboratory devices and external services. The cloud is also where artificial intelligence and human intelligence cooperate. We depict this situation in Figure 6.

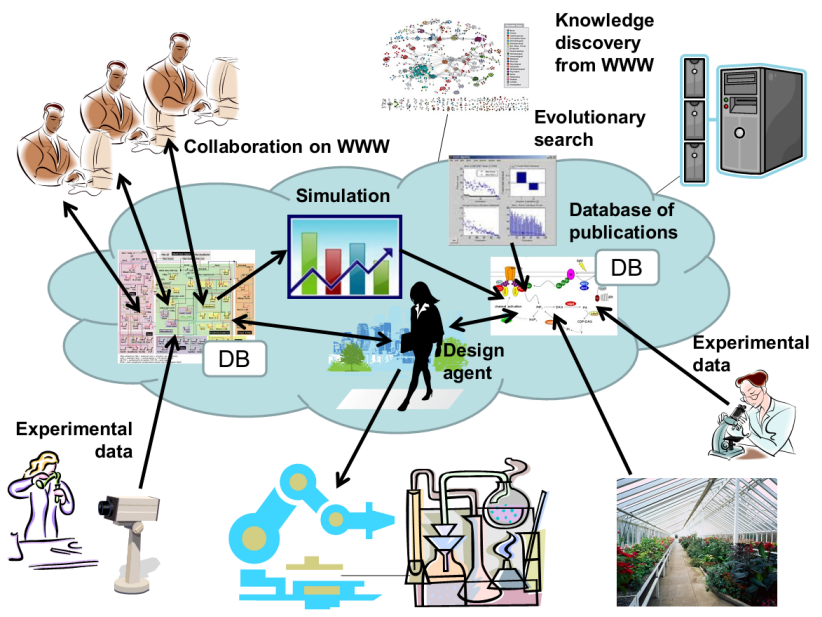

Fig. 6. The cloud

\section{Future of Molecular Robots}

We have described the vision that under the cloud, life and information evolve together. However, the interface between life and information is somewhat largescale and is often subjected to human intervention. More direct interfaces between the two are expected in the future.

Murata et al. recently summarized the evolution scenario of molecular robots [9. They explain the evolution of molecular robots in several stages. In the current (0th) stage, single-molecular robots like DNA spiders have been constructed, but their movement is heavily ruled by Brownian motion. In the first stage, in order to control or utilize the randomness of molecular reactions, compartments such as artificial membranes will be created, leading to amoebatype molecular robots. In the second stage, slime-type robots, whose size will 
reach the millimeter scale, will utilize the three-dimensional chemical wave field in a gel for movement. In the third stage, multi-cellular molecular robots will be created, and in the fourth stage and on, hybrid molecular robots that directly interface with electronic computers will emerge.

\subsection{Connecting Chemical-Biological and Electronic Systems}

Although foreseen in the last stage of the evolution of molecular robots, directly connecting or even merging chemical-biological and electronic systems has already become one of the biggest trends in science and technology, including BMI (brain-machine interface), MEMS (micro-electro-mechanical system), ultrafast DNA sequencing, and bio-imaging. Molecular robots are expected to solve many problems currently faced by human society. We believe that molecular robotics should participate in this trend as early as possible.

Electronic computers are thought to face a limitation in terms of miniaturization. Molecular electronics was once regarded as a key technology in construction of nanometer-scale circuits. However, the problem of how to connect molecular transistors with themselves or silicon circuits remains. Although DNA nanotechnology (DNA origami) was expected to solve this problem, there remain technical obstacles to overcome 6 .

In contrast, the miniaturization technology of VLSI has progressed, and currently, the so-called 20nm-process is used for production. Electronic computers now face an energy dissipation problem, which is closely related to computation errors, because a reduction in energy leads to a loss of exactness with each switching. Further miniaturization should also increase error probability.

\subsection{Dream}

In this era of parallel computing, the fastest supercomputers are composed of a huge number of many-core nodes connected by ultrafast networks. What types of application are run on such supercomputers? Scientific simulation remains one of the most important applications, but more computing resources are used for machine learning, searching and optimization, in which evolutionary computation is also included. These types of computers tolerate errors at some level. In reality, parallel computation often reports errors in both hardware and software.

Therefore, error-tolerant computation, such as neural networks, are likely the key to future computers. Thinking about neural networks, we are tempted to make an analogy with the brain, where computation is conducted by the transmission of electric pulses of neurons, while learning takes place through changes in synaptic connections, which are molecular in nature and very slow compared to computation.

With this analogy in mind, we propose the architecture (or dream) of a molecular-electronic chip. This consists of neurons composed of VLSI circuits that are laid out on one side of the chip. Neurons contain terminals that go pass through to the other side of the chip, which is immersed within a solution in which molecular robots move. One side of the chip is dry while the other is 
wet. Molecular robots may stick to some terminals and transmit electric current themselves or put in place molecules to connect terminals. Computation is conducted by electronic current along the circuits and connections between terminals, while learning is carried out by molecular robots. Note that this chip also realizes the trend of merging chemical-biological and electronic systems.

We can generalize the above dream and foresee a future in which molecular robots gain the ability to evolve. As they become a part of electronic computers, both the information stored therein and the computers themselves evolve. In addition, molecular robots interface with cells. This means that life and artificial life evolve together. Therefore, co-evolution of information, life and artificial life will become possible, and will lead to a new stage of intelligence, while the borders among the three will eventually disappear.

Open Access. This chapter is distributed under the terms of the Creative Commons Attribution Noncommercial License, which permits any noncommercial use, distribution, and reproduction in any medium, provided the original author(s) and source are credited.

\section{References}

1. Bath, J., Turberfield, A.J.: DNA nanomachines. Nat. Nanotechnol. 2(5), 275-284 (2007)

2. Channon, K., Bromley, E.H.C., Woolfson, D.N.: Synthetic biology through biomolecular design and engineering. Curr. Opin. Struct. Biol. 18(4), 491-498 (2008)

3. Cho, E.J., Lee, J.W., Ellington, A.D.: Applications of aptamers as sensors. Annu. Rev. Anal. Chem. 2, 241-264 (2009)

4. Kawamata, I., Hagiya, M.: (2012), http://hagi.is.s.u-tokyo.ac.jp/syn-biol/

5. Kawamata, I., Tanaka, F., Hagiya, M.: Automatic Design of DNA Logic Gates Based on Kinetic Simulation. In: Deaton, R., Suyama, A. (eds.) DNA 15. LNCS, vol. 5877, pp. 88-96. Springer, Heidelberg (2009)

6. Kershner, R.J., Bozano, L.D., Micheel, C.M., Hung, A.M., Fornof, A.R., Cha, J.N., Rettner, C.T., Bersani, M., Frommer, J., Rothemund, P.W.K., Wallraff, G.M.: Placement and orientation of individual DNA shapes on lithographically patterned surfaces. Nat. Nanotechnol. 4(9), 557-561 (2009)

7. Khalil, A.S., Collins, J.J.: Synthetic biology: applications come of age. Nat. Rev. Genet. 11(5), 367-379 (2010)

8. Kitano, H.: Systems Biology: A Brief Overview. Science 295(5560), 1662-1664 (2002)

9. Murata, S.: Molecular robotics: A new paradigm for artifacts. New Generation Computing 31(1), 27-45 (2013)

10. Seeman, N.C.: Nanomaterials based on DNA. Annu. Rev. Biochem. 79, 65-87 (2010) 